\title{
The Role of Apoptosis in Sepsis-Induced Kidney Dysfunction
}

\author{
Jo A. Crum ${ }^{\mathrm{a}}$ and Dr. Victoria Del Gaizo Moore ${ }^{\mathrm{a}}$
}

Sepsis is a system-wide inflammatory response to infection in the blood, which can be caused by any type of pathogen such as bacteria or fungi. Even though sepsis is the third highest cause of death in the world, only one third of Americans have heard of the condition. Such unawareness reflects the deficit in knowledge about how the body responds to sepsis. Accordingly, most current treatments for sepsis address symptoms rather than the cause. The initial response to sepsis is pervasive inflammation, mediated by cytokines secreted from immune cells. The massive amounts of cytokines release during sepsis undoubtedly affect the rest of the body, and immune cell dysfunction leads to not only the inability to fight the initial infection but increased propensity to secondary infection as well. During severe sepsis, end-organs often shut down causing irreparable damage and consequently organ failure is the primary cause of death in septic patients. How major organs, such as the kidneys, become dysfunctional during sepsis remains unclear. It is evident, however, that apoptosis is responsible for the impaired immune cell response during sepsis, and apoptosis is also implicated in damage to dendritic cells and tubular cells, and caspases may be involved specifically in tubular renal cell death. Therefore apoptosis may also be responsible for the decline of renal function during sepsis.

Keywords: Sepsis, Apotposis, BCL2, Acute Kidney Injury, Inflammation, Immunity

\section{Pathways of Cell Death}

\section{Background}

Cell signaling and protein-protein interactions are of particular importance when examining the biochemical basis of disease. Often, disease results from deregulation of normal cellular processes; one classic paradigm is uncontrolled cell death, either too much or not enough. In fact, uncontrolled cell death is a common theme in many different disease models. Therefore, understanding the process of cell death in the context of maintaining homeostasis is essential to unlocking mechanisms of disease.

There are three main classifications of cell death: apoptosis, autophagy, and necrosis. Each category is defined based on morphology of the dying cell, as well as tissue location and the dependence on lysosomes (1). Apoptosis, otherwise known as programmed cell death, is a means of keeping cell growth under control, killing and breaking down old and/or damaged cells, and serving as a regulatory process for both population control and viability of tissues in the body. Without apoptosis, there would be an excess of dysfunctional and potentially harmful cells in the body. Therefore, apoptosis is a normal part of biological processes. Billions of cells die in our bodies each day, and are either sloughed off to be excreted with other waste or broken down and used for parts through apoptosis (2). For example, during embryogenesis, apoptosis triggers death of the skin cells between fingers in the developing fetus resulting in individual digits (3). However, apoptosis can also contribute to the pathophysiology of disease when there is an imbalance of proteins and/or cellular signals.

Autophagy is a catabolic process of cell self-degradation. Like apoptosis, autophagy is an evolutionarily conserved mechanism that plays a role in the normal turnover of proteins and organelles, and thus both play a role in cellular homeostasis (1). However, autophagy is involved in the cell's response particularly to stress and starvation. Under nutrient starvation conditions, autophagy is a means to recycle cellular components so that the cell can survive this temporary stress. Conversely, extensive periods of nutrient withdrawal or degradation of pro-survival cellular factors can result in this form of non-apoptotic programmed cell death (4).

Sometimes referred to as 'accidental cell death,' necrosis is the death of a cell due to physical damage, such as inflammation following tissue damage, or other external factors. During necrosis, the cell does not destroy itself it merely dies (5). Unlike apoptosis and autophagy, no part of necrosis is organized or managed; rather, it is simply cells dying because of physical stress. Also unlike apoptosis and autophagy, necrosis in not an evolutionarily conserved mechanism and multiple cells die all at once during necrosis.

In wide-spread cell death, the distinction between the apoptosis and necrosis, as well as apoptosis and autophagy, is not always clear cut. In fact, it has been suggested that components from all three mechanisms work together in some instances (1) (Figure 1). Autophagy and apoptosis are apparently linked by crosstalk, since both pathways are regulated by some of the same common factors and proteins (7 and 8). Conversely, cellular signals that induce apoptosis activation induce autophagy, whereas signals that inhibit apoptosis also inhibit autophagy (9).

The three types of cell death, however, have very distinct visible cell patterns while cell death is occurring, making discrimination easier if the dying cell itself can be observed. Apoptosis is characterized by cytoplasmic and nuclear condensation, DNA fragmentation, membrane blebbing, and lysosomal degradation of apoptotic bodies by phagocytes (6). Apoptotic cells are rapidly engulfed without the loss of cellular material and therefore the process does not provoke an inflammatory response. 


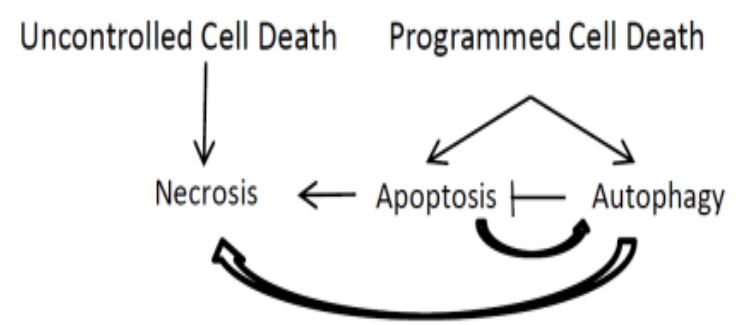

Figure 1: The interplay between programmed and uncontrolled cell death. All three types of cell death can affect at least one of the others, thus deciphering which mechanism(s) are involved in a particular pathophysiology can be difficult.

On the flipside of orderly cell death, autophagy is not associated with phagocytosis but is characterized by the presence of distinct vacuoles that fuse with lysosomes within the dying cells. It is in these autophagic vacuoles that self-degradation occurs (1).

Since necrosis is a response to damage of the cellular membrane by external sources, an influx of water and extracellular ions flood the cell causing swelling to the point where the cell itself, along with the internal organelles, rupture (5). Contents of organelles, including cytoplasmic and lysosomal enzymes, are released into the extracellular fluid after the cell ruptures. In turn, a strong inflammatory response from nearby cells ensues, which can result in the death of nearby cells (6). For this reason, it is important to note that necrosis usually occurs in a trend-like fashion and can be haphazard, compared with apoptosis or autophagy, which occurs cell by cell.

During sepsis, autophagy is likely to occur since it has been observed during infections such as cystic fibrosis (10) and aids in immune cell regulation (11). However, apoptosis or necrosis are more probable causes for end-organ decline because of the vast inflammation, infection, and overall physiological stress incurred during severe sepsis. It is possible that both apoptosis and necrosis occur in different tissues for different reasons, and that apoptotic cells not cleared breakdown via secondary necrosis. Given that kidney function is vital and that kidney failure occurs during severe sepsis, determining the mechanism of kidney cell death is an important first step to uncovering preventive measures.

\section{Mechanisms of Apoptotic Cell Death}

Apoptosis has two main types of triggers: extracellular influences and intracellular influences. Extracellular influences can be toxins, cytokines, hormones, or growth factors. When these extracellular chemicals trigger a series of responses leading to cell death, the extrinsic pathway is said to be activated. These extracellular molecules bind to 'death receptors', which were named so because of the role they play in activating the death pathway within the cell. One example of a death receptor is FAS, which is held into place on the external surface of the cell membrane by adapter proteins such as FAS-associated death domain proteins (FADD) (12). The intracellular portion of the FAS membrane receptor is also associated with an inactive, or Pro- form, of a cysteinedependent aspartate-directed protease (caspase), Pro-caspase 8. Once FAS associates with an appropriate FAS ligand, a cascade of intracellular changes is initiated, beginning with cleavage of Pro-caspase 8 to catalytically active caspase 8 . Activated caspases have their own proteolytic activity and therefore cleave other proteins enzymatically digest proteins via hydrolysis. As a result, the cell is dismantled in a trickledown manner, as opposed to the dead cell body remaining intact causing inflammation, which occurs during necrotic cell death $(13,14)$. One target of caspase 8 is Pro-caspase 3, resulting in activated caspase 3 which can then cleave other cellular proteins. Another target is a pro-apoptotic protein, BH3 interacting domain death agonist (BID) that also needs to be cleaved to become activated. Activated BID then travels to the outer membrane of the mitochondria to induce apoptosis (see below).

The intrinsic pathway does not commence in the same manner. As its name implies, the intrinsic pathway starts with intracellular signals resulting from internal damage to the cell, such as DNA damage or other events that negatively impacts the integrity of the cell (5). These signals cause the cessation of protein production for anti-apoptotic messages as well as the transcriptional upregulation of pro-death molecules, which migrate to the outside of the mitochondria. Then, apoptosis is invoked because pores form in the outer mitochondrial membrane, termed Mitochondrial Outer Membrane Permeability (MOMP) (15). MOMP allows proteins such as cytochrome $c$, which is normally retained within mitochondria and participates in ATP synthesis by shuttling electrons, to be released into the cytosol. Consequently, cytochrome $c$ release is considered the point of no return (16) as the lack of cytochrome $c$ in the mitochondria impedes generation of any further ATP. Additionally, once the cell reaches this point, caspases are activated but have no way of becoming deactivated, thus there is no return to homeostatic conditions. Cytochrome $c$ along with APAF-1, another mitochondrial factor released during MOMP, forms a complex with caspase 9 that then activates caspase 3 (17) (Figure 2).

A common mechanism of both the extrinsic and intrinsic pathways is the use of caspases. The initiator caspases for each are different, Caspase- 8 to cleave BID or directly cleave caspase 3 in the extracellular pathway or caspase 9 for the intracellular pathway. However, in either case caspase 3 acts as an executioner caspase and both pathways converge at the mitochondria. 


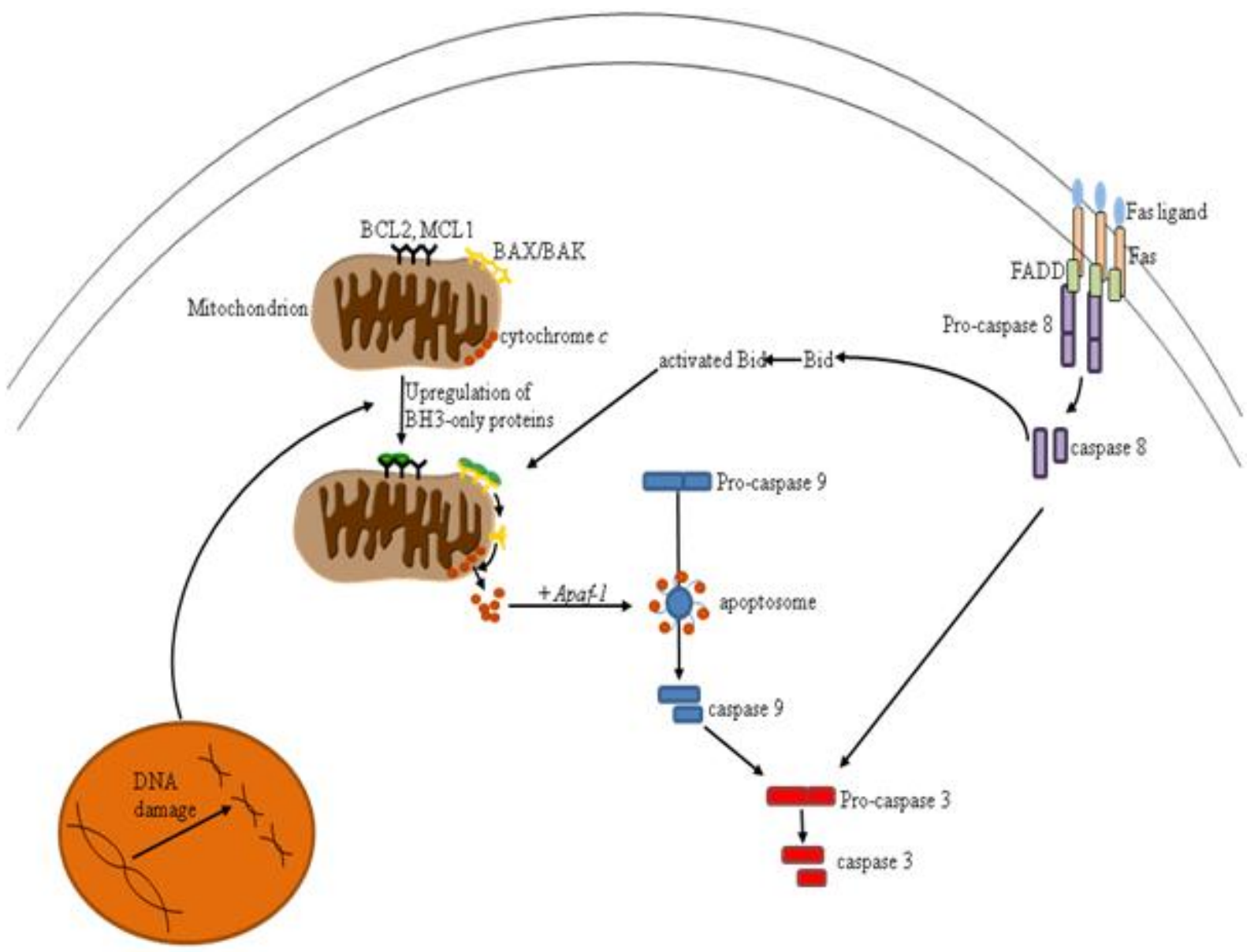

Figure 2: The extrinsic and intrinsic apoptotic pathways. Either external signals (right hand side of figure) cause activation of initiator caspase 8, or internal signals (left hand side) cause activation of initiator 9. Eventually, both pathways lead to MOMP at the mitochondria and the unleashing of executioner caspase 3.

During sepsis, immune cells (18), renal tubular cells (19), and vascular endothelial cells (20) die via apoptosis. Kidney cells are exposed to high levels of circulating cytokines, invading pathogens due to the primary and possibly secondary infections, as well as other extracellular stresses. Therefore, apoptosis could also be responsible for kidney damage during sepsis.

\section{BCL2 family of Proteins}

Cellular homeostasis is largely controlled by the balance of pro-survival and pro-death proteins, and various diseases arise when deviation from the norm occur (21). The major class of pro-survival and pro-death proteins, also referred to as antiapoptotic and pro-apoptotic proteins, belongs to the BCL2 family, whose name is derived from a B-cell Lymphoma where the initial protein and namesake of the family, BCL2, was first discovered (22-24). A well accepted concept is that apoptosis occurs when the balance of these proteins tips toward death, changing the mitochondrial membrane as discussed above. After MOMP, cytochrome $c$ release and caspase 3 become activated, destructive activities such as condensation of chromatin and exposure of phosphotidyl serine (PS) on the outer leaflet of the cell membrane, a signal that tags the dying cell for phagocytosis, transpire. Other effects created by pro-apoptotic signals are increased intracellular reactive oxygen species, the unfolding of proteins, and the cessation of growth factors (25). These all are indicators of a cell heading down the pathway towards apoptosis.

When apoptosis does occur at an unbalanced rate, anti- and pro- apoptotic proteins are of particular importance, especially since deviations from the norm have been linked to When apoptosis does occur at an unbalanced rate, the proteins that are pro-survival or pro-death various diseases (21). The major class of these proteins belongs to the BCL2 family, whose name is derived from a B-cell Lymphoma where the initial protein and namesake of the family, BCL2, was first discovered (22-24). A well accepted concept is that apoptosis occurs when the balance of these proteins tips toward death, changing the mitochondrial membrane as discussed above. After MOMP, cytochrome $c$ release and caspase 3 become activated, destructive activities such as condensation of chromatin and exposure of phosphotidyl serine (PS) on the outer leaflet of the cell membrane, a signal that tags the dying cell for phagocytosis, transpire. Other effects created by proapoptotic signals are increased intracellular reactive oxygen species, the unfolding of proteins, and the cessation of growth 
factors (25). These all are indicators of a cell heading down the pathway towards apoptosis.

The necessary component for a protein to be included in the BCL2 family is the presence of at least one BCL2 protein homology domain. This domain allows the protein to interact intermolecularly with other proteins to control apoptosis (26).
Anti-apoptotic proteins are necessary for cell survival and function, and have up to four BCL2 homology domains (Table 1 and Figure 3). Pro-Apoptotic proteins include BAX and BAK, which have 3 homology domains, as well as 'BH3only' proteins - named aptly due to the presence of just the BCL2 homology domain (Table 1).

Table 1: Classification of BCL2 family proteins.

\begin{tabular}{ccc}
\hline & Anti-apoptotic & Pro-apoptotic \\
\hline Multidomain & $\mathrm{BCL}, \mathrm{BCL}-\mathrm{X}_{\mathrm{L}}, \mathrm{BCL}-\mathrm{W}, \mathrm{MCL1}, \mathrm{BFL1}$ & $\mathrm{BAX}$ and BAK \\
BH3-only domain & $\mathrm{BAD}, \mathrm{BIK}, \mathrm{NOXA}, \mathrm{PUMA}, \mathrm{BMF}$, and HRK & BIM and BID \\
\hline
\end{tabular}

Multi-domain pro-apoptotic BAX and BAK are unique in their function as well as their composition, and as such are the only pro-apoptotic proteins able to directly trigger permeabilization of the mitochondrial membranes. BH3-only proteins are also unique in that they can either bind and inhibit anti-apoptotic proteins, or directly bind BAX/BAK so they may carry out their functions leading to cell death,(Figure 3) $(15,26)$. It is important to note that these proteins are not the only ones involved in apoptosis, rather they are the most well-known and characterized.

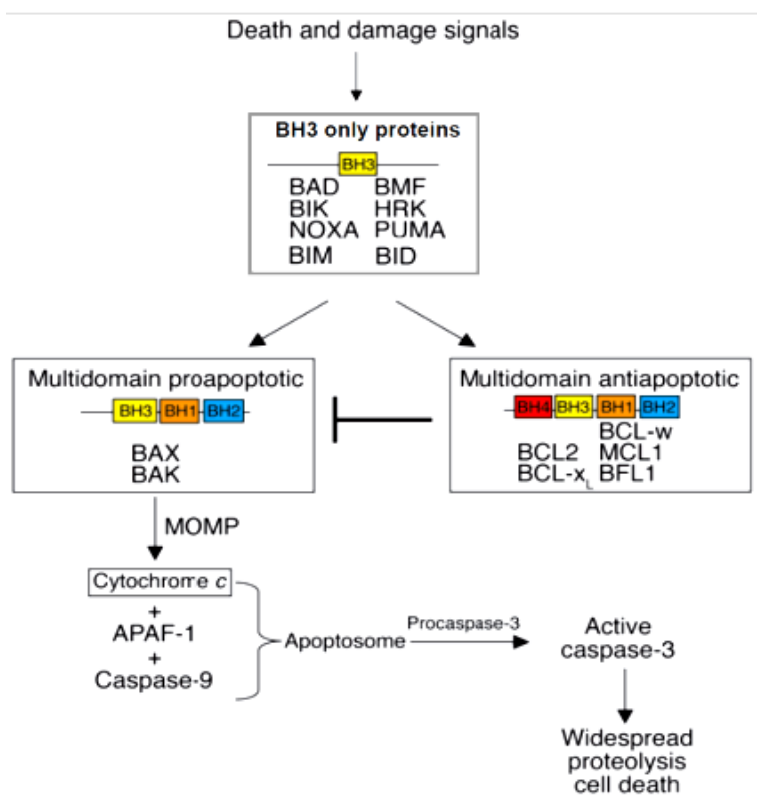

Figure 3: Depiction of BCL2 proteins' control of apoptosis.

After certain pro-death signals are induced, upregulation of $\mathrm{BH} 3$ only proteins either directly cause activation of $\mathrm{BAX} / \mathrm{BAK}$ to induce cytochrome $c$ release or bind to multidomain antiapoptotic protein to trigger MOMP. Once cytochrome $c$ is released into the cytosol, it combines with APAF-1 and caspase-9 in a complex know as the apoptosome, which is able to cleave Pro-caspase 3 thereby activating its proteolytic activity to cleave other cellular proteins (Adapted from 26).

If apoptosis is indeed occurring in end-organs such as the kidneys during sepsis, the most certainly there would be involvement of BCL2 family proteins. As such, changes in
BCL2 protein expression would be observable. In fact, immune cell apoptosis is characterized by upregulation of BIM (18) and overexpression of BCL2 improves survival in a sepsis animal model (27).

\section{Apoptosis in Disease}

The balance of these pro-survival and pro-death proteins is what regulates apoptosis in cells - determining life or death. When the balance is not maintained, problems within the cell arise. When one cell is affected, damage is negligible, but unfortunately almost never is only one cell affected by disruption to a system. Two relevant examples of extreme disruptions of apoptosis are cancer and cardiomyopathy. In cancer, cells fail to undergo apoptosis, becoming immortal and continue to divide rather than dying (28). In this case, pro-survival BCL2 proteins have been found in higher than normal levels, playing a key role in the persistence of cancer cells. As a counter-example, too much apoptosis occurs after a myocardial infarction such as a heart attack, or stroke. In these cases, damage occurs upon re-oxygenation to the heart causing activation of the caspase cascade and premature apoptosis. As a result, this premature activation causes lots of cell death. The possibility of both extremes adds to the intrigue of studying the balance and interaction of these proteins in maintaining homeostasis, but also adds to the complexity and delicate nature of this biological process.

In theory, it seems as if the most logical way of handling these conditions in which apoptosis is disrupted would be to simply induce or inhibit apoptosis. Cell death, however, is not as simple as 'turn on $\mathrm{x}$ ' or 'turn off $\mathrm{y}$ '. There are large numbers of proteins and factors involved in apoptosis, and very similar pieces that can activate other types of cell death. Inducing cell death is technically much easier to accomplish, due to a number of pathways that are fairly easy to cause death, such as stress in the form of heat. Preventing cell death is complicated due to the nature and number of protein interactions involved in deactivating pro-death stimuli and the ease of activating death. Even if one were able to interrupt the pathway of apoptosis, it is possible that certain functions had taken place which already pushed the cell past the point of repair. Hence, identifying the mode of cell death in septic tissue is key not only to further understanding the disease but is also necessary if preventative measures are to be found. 


\section{Sepsis}

\section{Background}

Sepsis, systemic inflammation that occurs by infection with gram positive or gram negative bacteria or fungi, is one of the leading causes of emergency room death in the United States and affects more than 750,000 Americans each year (29-31). Initial stages of sepsis are pro-inflammatory responses to infection often described as a 'cytokine storm' $(30,32)$. There is neither one cause nor a specific phenotype of symptoms; however initial clinical presentation often includes fever, elevated white blood cell count, abnormal body temperature, abnormal heart rate, and abnormal respiration rate (33). Clinically, sepsis can be thought of as a continuum, commencing with infection and ending in death. In the initial stages of sepsis, the body tries to fight the infection with a pro-inflammatory reaction. However, if this stage persists too long, a shift towards an anti-inflammatory and immunosuppressive state occurs, causing patients to be more susceptible to a secondary infections and organ dysfunction (30). Ultimately, if multiple organs fail and hypotension develops, recovery is not likely. Despite advances in therapeutic strategies to minimize or evade early sepsis complications, a reduction in mortality has not been obtained (34). Therefore, understanding the role of innate and adaptive immune response, as well as mechanisms of cell death, in end-organs during sepsis could potentially lead to more effective treatments and/or preventions.

\section{The role of the immune system in sepsis}

The immune system response to pathogenic organisms is mediated by both the innate and adaptive arms of cellular immunity. These systems work in concert, with innate immunity constitutively present and therefore reacting immediately to infection, while adaptive immunity takes time to respond and develop. As such, the innate response is more generalized while the adaptive response is very specific to the invading pathogen. Specialized immune cells such as dendritic cells, neutrophils, phagocytes, macrophages (4), and natural killer (NK) cells (35) mediate the innate proinflammatory response. These cells are quick to respond, with neutrophils being the first to a site of infection during the early phases of sepsis to mount a response to neutralize the foreign invader. Macrophages, neutrophils, and dendritic cells are phagocytes that engulf bacteria or viruses and release reactive oxygen species, breaking down the pathogen's cellular components which can then be cleared by the body (35). Additionally, macrophages and NK cells release cytokines. Cytokines are proteins and polypeptides that act as chemical mediators of infection and are produced when the immune system is stressed from persistent and lasting inflammation $(36,37)$. Not only does the release of cytokines enhance the innate immune response, but also signals adaptive immune $\mathrm{B}$ and $\mathrm{T}$ cells to produce antibodies and secrete cytokines (35). Furthermore, dendritic cells present antigens from engulfed pathogens to T cells as well as secrete cytokines that enhance $T$ cell cytokine production.

Production of several cytokines, such as tumor necrosis factor alpha (TNF- $\alpha$ ), interleukin (IL) 1 beta (IL-1- $\beta$ ), and interferon gamma (IFN- $\gamma$ ), become consistently elevated in sepsis due to transcriptional upregulation of inflammatory genes (30). This upregulation results from the adaptive immune response that recognizes molecules derived from microbes that are presented, after phagocytosis during the initial innate immune response (38). Pro-inflammatory molecules such as these activate caspases specific to immune response $(32,38)$ (refer to Figure 2). TNF- $\alpha$ and IFN- $\gamma$ can also stimulate inducible nitric oxide synthase (iNOS) to produce nitric oxide (NO), a chemical that is also part of the body's immune defenses that forms free radicals that are toxic to bacteria $(38,39)$. Thus, an intricate signaling cascade is responsible for immune response to infection, and if the proinflammatory response is not carefully regulated, it could become detrimental. When the response to infection overcompensates, anti-inflammatory mechanisms commence leading to massive immune cell death and high levels of circulating pro-inflammatory cytokines. Large amounts of cytokines could very well stress tissues to a point where their regular function becomes inhibited. One likely response in these tissues to such a stress would be apoptosis.

\section{Sepsis and Apoptosis}

As described above, secretion of TNF- $\alpha$ is immense during the innate and adaptive immune response during infection leading into sepsis. TNF- $\alpha$ is one of the extracellular ligands that can trigger the extrinsic mitochondrial pathway of apoptosis (40). Furthermore, during sustained inflammatory response, IFN- $\gamma$ and TNF- $\alpha$ can trigger Fas-ligand production, another factor that mediates extrinsic apoptosis (Figure 2). Not surprisingly, once the initial immune response has been mounted and infection persists, immune cell numbers become drastically reduced due to extensive cell death via apoptosis (32, 41). Accordingly, pro-apoptotic BCL2 family proteins are upregulated in immune cells in both mouse models of sepsis and septic patients. Specifically, protein levels of proapoptotic BIM and PUMA are increased in splenocytes of septic mice and knocking down both decreases immune cell death (42). Furthermore, a dominant negative mutant of Fasassociated death domain or knocking out BID increases survival of septic mice, while BIM knockouts completely protect lymphocytes from apoptosis (43). Corroborating the mouse model data, blood samples from severe sepsis patients has confirmed accelerated apoptosis, caspase 3 activation and PS externalization in different lymphocyte populations (44). The authors of that study also found BCL2 protein levels decreased and BIM upregulated. Other studies have shown BCL2 transgenetic mice are resistant to sepsis-induced apoptosis and massive lymphocyte apoptosis was found in autopsies of septic patients (32). Induction of wide-spread apoptosis in immune cells could be a major avenue for the progression of sepsis to severe sepsis with organ damage. A decrease in the number of critical response cells, such as dendritic cells, $\mathrm{T}$, and $\mathrm{B}$ cells, would inevitably allow inflammation to persist and negatively impact the function of multiple other tissues.

As alluded to, sepsis and severe sepsis can be experimentally induced using mouse models, allowing closer analysis of the molecular mechanisms of the disease. One of the more popular ways to induce sepsis in experimental disease models is intraperitoneal injection of lipopolysaccharide (LPS) into mice, simulating the LPS exposure during a bacterial infection (32). While much research about immune cell death has arisen from such studies, information about end-organ cell death has also been 
revealed. In particular, the release of pro-inflammatory cytokines TNF- $\alpha$ and IL1- $\beta$ has been found to turn on a secondary inflammatory cascade by activating nuclear factor kappa beta $(\mathrm{NF}-\kappa \mathrm{B})$, a transcription factor that produces further inflammatory cytokines. Greten et al pointed out the negative regulator relationship between NF- $\kappa \mathrm{B}$ and IL1- $\beta$ secretion in their 2007 study (45). IKK $\beta$, an activator of NF$\kappa \mathrm{B}$, can be used to block transcription of subsequent inflammatory chemicals. However, this would not be an ideal therapeutic treatment since $\mathrm{NF}-\mathrm{kB}$ is a principle player of the host's defense system; removal of this activating piece could make the patient more susceptible to infection as well as increasing susceptibility to apoptosis. Furthermore, even though a IKK $\beta / N F-\kappa B$ blockade could stop the secondary release of further inflammatory signals in theory, such a block may not be effective. For example, to compensate for a lack of secondary release caused by an IKK $\beta / \mathrm{NF}-\kappa \mathrm{B}$ blockade, the body could produce more inflammatory cytokines in an attempt to trigger the activation of the secondary cascade, rather than ceasing production of the same inflammatory cytokines thereby increasing apoptosis. In fact, silencing of $\mathrm{NF}-\kappa \mathrm{B}$ proved to be successful in providing supporting evidence as to the importance of IKK $\beta$ and NF- $\kappa \mathrm{B}$ in the proinflammatory pathway involved in sepsis and the triggering of major organ failure (45). Since NF- $\mathrm{KB}$ can be upregulated by TNF- $\alpha$, this data also supports the notion that the external apoptotic pathway could be induced in organ failure.

\section{Kidney Damage Induced By Sepsis}

Although the mortality rate of patients with sepsis has decreased slightly in recent years, the mortality of patients with septic shock and/or Acute Kidney Injury (AKI) caused by sepsis remains high. AKI, which is characterized by rapid loss of kidney function, is caused by excessive kidney cell death in response to cytokines released as part of the body's immune response $(26,30)$. While it is clear that during sepsis apoptosis kills immune cells on a large scale, the role of apoptosis in damage to end-organs, such as the kidneys, is relatively unknown (30).

Some insight about Sepsis Induced AKI (SI-AKI) comes from analysis of increased levels of kidney cell NO levels during severe sepsis. High levels of systemic TNF- $\alpha$ was found to amplify activity of iNOS, which in turn produced more NO and swinging the system balance towards cytotoxic levels, particularly in kidney cells (46). While it was known that NO could be produced by tubular endothelial cells, this study demonstrated that NO could induce apoptosis independently of INF- $\gamma$ by activating Caspase- 8 .

More evidence supporting the importance of the caspase family in the pathogenesis of kidney injury comes from a study using a rat AKI model. AKI rats were given the pancaspase inhibitor benzyloxycarbonyl-Val-Ala-Asp-flu-oromethylketone (Z-VAD-FMK); protection from renal function was found to be significantly higher in inhibitor treated vs. untreated AKI rats (47). This particular experimental study further supports the involvement of caspases in the mechanism of cell death in SA-AKI, and looks into potential interventional treatments that target the mechanism of disease rather than the symptoms for future medical use. In a very recent study conducted by Rodrigues et al, continuous erythropoietin receptor activator (CERA) was administered as a pre-treatment before undergoing cecal ligation and puncture (CLP) to observe the effects of induced sepsis, SA-AKI, and tubular dysfunction (48). It is known that toll-like receptors (TLR's) are important microbial pathogen receptors on innate immune cells and a specific TLR, TLR4, is found in higher quantities in the kidneys of septic animals (49). TLR4 stimulates the production of $\mathrm{NF}-\kappa \mathrm{B}$, which induces systemic cytokine production during septic shock (50). Silencing of $\mathrm{NF}-\kappa \mathrm{B}$ genes in mice creates resistance to septic shock and related mortality, supporting the involvement of inflammatory pathways (48). NF- $\kappa \mathrm{B}$ also has vasopressor, anti-apoptotic, anti-inflammatory, and immunomodulatory activities that can reduce oxidative stress and lipid peroxidation (48). In the Rodrigues et al study, plasma levels of IL-1 $\beta$, IL-2, IL-6, IL10, IFN- $\gamma$, and TNF- $\alpha$ were quantified 24 hours after CLP. Cytokine levels were higher in CLP than controls, but CLP+CERA treated rats were more comparable to controls in spite of still having elevated levels in IL- $1 \beta$, IFN- $\gamma$, and TNF$\alpha$. Overall, CERA administration was found to decrease liver damage, protect renal function, and protect glomerular filtration against sepsis-induced functional decrease and preserved urinary output (48). Use of this model, which nicely mimics polymicrobial-triggered sepsis experienced in the clinical setting, suggests possible follow-up studies that would include addition of vassopressors, antibiotics, and/or other intervention strategies. Furthermore, animal models of sepsis, such as CLP or LPS injection, could be used in evaluating the mechanism of SI-AKI and possible interventions.

\section{Conclusions}

Cellular death is part of, and contributes to, many pathological conditions. The type of cell death involved sometimes is clear, while other times it is not. Understanding each type of cell death at the molecular and biochemical level is important in elucidating their roles in different instances. Apoptosis, in particular, has been clearly linked to many diseases including sepsis. During sepsis, immune cell dysfunction arises from excessive loss of immune effectors cells via apoptosis, leading to the inability to clear infection thus rendering the body susceptible to subsequent infections (25). Consequently, serious secondary infections and complications lead to irreparable damage of end-organs and sometimes death. How end-organs, such as the kidney, fail at a cellular level has not thoroughly been uncovered, and particularly during SI-AKI. Given the role of cytokines during sepsis such as TNF- $\alpha$, which can trigger extrinsic apoptosis, as well as secondary infection, and generalized stress on the body during sepsis, probable mechanisms of kidney cell death are necrosis or apoptosis. Initial experimental evidence supports apoptosis, however much more research is necessary to definitively conclude it is the sole source of cell death. Therefore, much research investigating the role of apoptosis in severe sepsis is underway in hopes that some insight from studying the proteins involved in triggering cell death may be provided. Once revealed, this knowledge will not only contribute to understanding of the intricacies' of sepsis, but also render information for prevention and possible therapeutic intervention. 


\section{References:}

1. Thorburn A. Apoptosis and autophagy: regulatory connections between two supposedly different processes. Apoptosis. 2008 Jan; 13(1):1-9.

2. Vaux DL, Korsmeyer SJ. Cell death in development. Cell. 1999 Jan; 96(2):245-54.

3. Zuzarte-Luis, V and Hurle, JM. "Programmed cell death in the developing limb." Int J Dev Biol. 2002 46(7): 871-6.

4. Hsieh YC, Athar M, Chaudry IH. When apoptosis meets autophagy: deciding cell fate after trauma and sepsis.Trends Mol Med. 2009 Mar;15(3):129-38.

5. Differences between necrosis and apoptosis. Cell DeathApoptosis and Necrosis. [online] http://www.rocheappliedscience.com/PROD_INF/MANUALS/CELL_MAN/apo ptosis_003_004.pdf

6. Charras GT. A short history of blebbing. J Microsc. 2008 Sep;231(3):466-78.

7. Marquez RT, Xu L. Bcl-2:Beclin 1 complex: multiple, mechanisms regulating autophagy/apoptosis toggle switch. Am J Cancer Res. 2012 Feb 2(2):214-21.

8. Djavaheri-Mergny M, Maiuri MC, Kroemer G.Cross talk between apoptosis and autophagy by caspase-mediated cleavage of Beclin 1. Oncogene. 2010 Mar 25;29(12):1717-9.

9. Mizushima N, et al. Autophagy fights disease through cellular self-digestion. Nature 2008 Feb 451: 1069-1075.

10. A. Luciani, V. R. Villella, S. Esposito et al. Cystic fibrosis: a disorder with defective autophagy. Autophagy 2011 Jan 7(1): 104-106.

11. H. W. Virgin and B. Levine. Autophagy genes in immunity. Nature Immunology 2009 Jan 10(5): 461470.

12. Weinlich R, Dillon CP, Green DR. Ripped to death. Trends Cell Biol. 2011 Nov; 21(11):630-7.

13. Campbell NA, Reece JB, Urry LA, Cain ML, Wasserman SA, Minorsky PV, Jackson RB. Biology. San Francisco: Pearson. 2008. 224-25p.

14. Pathway Central: Cellular Apoptosis Pathway. SABiosciences. [online] http://www.sabiosciences.com/pathway.php?sn=Cellular Apoptosis_Pathway

15. Del Gaizo Moore V, Letai A. BH3 profiling - Measuring integrated function of the mitochondrial apoptotic pathway to predict cell fate decisions. Cancer Lett. 2012 Jan 8 (published online).

16. Von Ahsen O, Waterhouse NJ, Kuwana T, Newmeyer DD, Green DR. The 'harmless' release of cytochrome c. Cell Death Differ. 2000 Dec; 7(12):1192-9.

17. Brunelle JK, Letai A. Control of mitochondrial apoptosis by the Bcl-2 family. J Cell Sci. 2009 Feb 15; 122(Pt 4):437-41

18. Hotchkiss RS, Nicholson DW. Apoptosis and caspases regulate death and inflammation in sepsis. Nat Rev Immunol. 2006 Nov; 6(11):813-22.

19. Messaris E, Memos N, Chatzigianni E, Kataki A, Nikolopoulou M, Manouras A, Albanopoulos K,

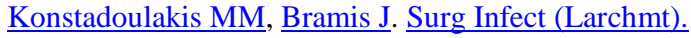
2008 Jun; 9(3):377-88.

20. Matsuda N, Teramae H, Yamamoto S, Takano K-I, Takano $\mathrm{Y}$, and Hattori Y. Increased death receptor pathway of apoptotic signaling in septic mouse aorta: effect of systemic delivery of FADD siRNA. AJP Heart 2010 Jan; 298(1): H92-H101

21. Del Gaizo Moore V, Letai A. Rational design of therapeutics targeting the BCL-2 family: are some cancer cells primed for death but waiting for a final push? Adv Exp Med Biol. 2008; 615: 159-75.

22. Bakhshi A, Jensen JP, Goldman P, Wright JJ, McBride OW, Epstein AL, Korsmeyer SJ. Cloning the chromosomal breakpoint of $\mathrm{t}(14 ; 18)$ human lymphomas: clustering around $\mathrm{JH}$ on chromosome 14 and near a transcriptional unit on 18. Cell $1985 \mathrm{Jul}$; 41: 899-906.

23. Cleary ML, Sklar J. Nucleotide sequence of a $t(14 ; 18)$ chromosomal breakpoint in follicular lymphoma and demonstration of a breakpoint-cluster region near a transcriptionally active locus on chromosome 18, Proceedings of the National Academy of Sciences of the United States of America 1985 Nov; 82: 7439-7443.

24. Tsujimoto Y, Gorham J, Cossman J, Jaffe E, Croce CM. The $\mathrm{t}(14 ; 18)$ chromosome translocations involved in $\mathrm{B}$ cell neoplasms result from mistakes in VDJ joining. Science 1985 Sep; 229: 1390-1393.

25. Hotchkiss RS, Strasser A, McDunn J, Swanson PE. Mechanisms of Disease: Cell Death. The New England Journal of Medicine 2009 Oct; 361: 1570-1583.

26. Certo M, Del Gaizo Moore V, Nishino M, Wei G, Korsmeyer S, Armstrong SA, Letai A. Mitochondria primed by death signals determine cellular addiction to antiapoptotic BCL-2 family members. Cancer Cell. 2006 May; 9(5):351-65.

27. Hotchkiss RS, Swanson PE, Knudson CM, Chang KC, Cobb JP, Osborne DF, Zollner KM, Buchman TG, Korsmeyer SJ, Karl IE. Overexpression of Bcl-2 in transgenic mice decreases apoptosis and improves survival in sepsis. J Immunol. 1999 Apr 1; 162(7):414856.

28. Binder C, Marx D, Overhoff R, Binder L, Schauer A, Hiddemann W. Bcl-2 protein expression in breast cancer in relation to established prognostic factors and other clinicopathological variables. Ann Oncol 1995 Dec; 6(10):1005-1010.

29. Levy MM, Fink MP, Marshall JC et al. SCCM/ESICM/ACCP/ATS/SIS international sepsis definitions conference. Intensive Care Medicine 2003 Apr; 29: 530-538.

30. Pelte CH, Cawla LS. Novel therapeutic targets for prevention and therapy of sepsis associated acute kidney injury. Current Drug Targets 2009 Dec; 10: 1205-1211.

31. Martin GS, Mannino DM, Eaton S, Moss M. The epidemiology of sepsis in the United States from 1979 through 2000. The New England Journal of Medicine 2003 Apr; 348: 1546-1554.

32. Apoptosis and caspases regulate death and inflammation in sepsis. Hotchkiss RS, Nicholson DW. Nat Rev Immunol. 2006 Nov; 6(11):813-22.

33. Bone RC, Balk RA, Cerra FB, Dellinger RP, Fein AM, Knaus WA, Schein RM, Sibbald WJ. Definitions for sepsis and organ failure and guidelines for the use of innovative therapies in sepsis. The ACCP/SCCM Consensus Conference Committee. American College of Chest Physicians/Society of Critical Care Medicine. Chest. 1992 Jun; 101(6): 1644-55. 
34. Rivers E, Nguyen B, Havstad S, Ressler J, Muzzin A, Knoblich B, Peterson E, Tomlanovich M; Early GoalDirected Therapy Collaborative Group. Early goaldirected therapy in the treatment of severe sepsis and septic shock. N Engl J Med. 2001 Nov 8; 345(19): 136877.

35. Chiche L, Forel JM, Thomas G, Farnarier C, Vely F, Bléry M, Papazian L, Vivier E. The role of natural killer cells in sepsis. J Biomed Biotechnol. 2011; 2011: article ID 986491.

36. O'Brien JM, Ali NA, Aberegg SK, Abraham E. Sepsis. American Journal of Medicine 2007 Dec; 120: 10121022.

37. Cinel I, Opal SM. Molecular biology of inflammation and sepsis: a primer. Crit Care Med. 2009 Jan; 37(1): 291304.

38. Jean-Baptiste E.Cellular mechanisms in sepsis. J Intensive Care Med. 2007 Mar-Apr; 22(2): 63-72.

39. Vandenabeele P, Declercq W, Van Herreweghe F, Vanden Berghe T. The role of the kinases RIP1 and RIP3 in TNF-induced necrosis. Sci Signal. 2010 Mar 30; 3(115): re4.

40. Venet F, et al. Regulatory $\mathrm{T}$ cell populations in sepsis and trauma. J Leukoc Biol 2008; 83: 523-535.

41. Brahmamdam P, Watanabe E, Unsinger J, Chang KC, Schierding W, Hoekzema AS, Zhou TT, McDonough JS, Holemon H, Heidel JD, Coopersmith CM, McDunn JE, Hotchkiss RS. Targeted delivery of siRNA to cell death proteins in sepsis. Shock. 2009 Aug; 32(2): 131-9.

42. Chang KC, Unsinger J, Davis CG, Schwulst SJ, Muenzer JT, Strasser A, Hotchkiss RS. Multiple triggers of cell death in sepsis: death receptor and mitochondrialmediated apoptosis. FASEB J. 2007 Mar; 21(3): 708-19.
43. Weber SU, Schewe JC, Lehmann LE, Müller S, Book M, Klaschik S, Hoeft A, Stüber F. Crit Care. Induction of Bim and Bid gene expression during accelerated apoptosis in severe sepsis. 2008; 12(5): R128.

44. Greten FR, Arkan MC, Bollrath J, Hsu L-C, Goode J, Miething C, Goktuna SI, Neuenhah M, Fierer J, Paxian S, et al. Cell 2007 Sep; 130(5): 918931.

45. Martin GS, Mannino DM, Eaton S, Moss M.The epidemiology of sepsis in the United States from 1979 through 2000. The New England Journal of Medicine. 2003 Apr; 348: 1546-1554.

46. Du C, Guan Q, Diao H, Yin Z, Jevnikar AM. Nitric oxide induces apoptosis in renal tubular epithelial cells through activation of caspase-8. Am J Physiol Renal Physiol 2005 Dec; 290: F1044-F1054.

47. Homsi E, Janino P, de Faria JBL. Role of caspases on cell death, inflammation, and cell cycle in glycerol-induced acute renal failure. Kidney Intl 2006 Mar; 69: 13851392.

48. Rodrigues CE, Sanches TR, Volpini RA, Shimizu MHM, Kuriki PS, Camara NOS, Seguro AC, Andrade L. Effects of continuous erythropoietin receptor activator in sepsisinduced acute kidney injury and multi-organ dysfunction. PLoS ONE 2012 Jan; 7(1): e29893.

49. El-Achkar TM, Huang X, Plotkin Z, Sandoval RM, Rhodes GJ, et al. Sepsis induces changes in the expression and distribution of Toll-like receptor 4 in the rat kidney. Am J Physiol Renal Physiol 2006 May; 290(5): F1034-1043.

50. O'Sullivan AW, Wang JH, Redmond HP. NF-kappaB and p38 MAPK inhibition improve survival in endotoxin shock and in a cecal ligation and puncture model of sepsis in combination with antibiotic therapy. J Surg Res 2009 Mar; 152(1): 46-53. 